\section{From the Desk of Editor in Chief}

It's a great pleasure and privilege that AKMMC journal has passed 10 years of its successful Publication. We are indebted to our contributory authors, readers at home and abroad, teachers and management of AKMMC for their continued support for this. We will continue our thrive for publishing it regularly with more input in its different sections and maintain a high standard without compromising its quality.

Editorial on "Prostate cancer: Importance of screening and Early Diagnosis" emphasizes the need for early diagnosis and screening for prevention of prostate cancer, the second most common cause of cancer, mostly in males.

Original Article on "Domestic Hygiene practices and factors that risks for childhood diarrhoea among under five children in isolated chars of Gaibandha, Bangladesh" showed that community based behavierial change on personal hygiene and sanitation can reduce the childhood diarrhea.

Article on "Comparison of Left Radial versus Femoral Approaches for Coronary Procedures in Patients with Previous Coronary Artery Bypass Grafts" revealed that coronary angiography for post CABG patients through left radial approach seems to be effective, not inferior in terms of contrast volume, procedure and fluoroscopy time and other clinical endpoints compared to femoral access.

Original article on "A prospective observational study on treatment evaluation in patients newly diagnosed with Squamous Cell Carcinoma of head and neck in Bangladesh" showed that Squamous Cell Carcinoma of head and neck region are mostly presented in advance stage in Bangladesh and the majority of the patients are treated with combination chemotherapeutic regimens. This needs importance of early preventive measures and diagnosis for better management.

Article on "Unmet Need of Family Planning among Married Women of Reproductive Age in Rural areas of Manikganj District" revealed Family planning is more of a health necessity than option. Family planning programe in Bangladesh has achieved remarkable success over past decade but still more is to be done.
Article on "A Study on Sutureless and glue-free conjunctival autograft in pterygium surgery in a tertiary medical college hospital" described a simple technique which is safe for pterygium surgery that can prevent potential adverse reactions encountered with foreign materials .

Article on "Issues on Vector Related Diseases Transmission: People's Perception \& Practices" emphasizes the importance of community awareness in terms of perceptions, knowledge and practices regarding vector borne diseases for prevention of vector borne diseases in the locality.

Article on "Knowledge And Risk Behavior On Hepatitis C Virus Infection Among Laboratory Technicians In a Selected Army Medical Unit" showed adequate programe on updating knowledge, strict use of gloves, sterile instrument is a must for sample collection and minimizing transmission of HCV infection.

Article on "Effectiveness of Ranolazine to Prevent Myocardial Injury During Elective Percutaneous Coronary Intervention" revealed that Ranolazine is significantly effective in reducing the periprocedural myocardial injury in elective PCI.

Article on "Microbiological Study of Diabetic Foot Ulcer" showed that most of this are caused by polymicrobial agents These are sensitive to limited broad spectrum antibiotics and very narrow range of sensitivity for some species. Proper diabetic control and care of foot is essential to prevent this dreadly condition.

Article on "Aetiology, Clinical Profiles, Laboratory Profile, Outcome and Prognostic Factors of Pediatric Acute Liver Failure: Experience at a Tertiary Hospital of Bangladesh" revealed that Wilson disease is the most common cause of acute liver failure in children Early diagnosis is essential for better prognosis. Majority of the viral etiology improved with supportive care.

Article on "Early Outcome of Lichtenstein Technique for Inguinal Hernia Repair In Tertiary Care Hospital" showed that this technique is easy, simple and very rationale for inguinal hernia repair and the recurrence rate is low. 
From the Desk of Editor in Chief

Article on "An Observational Study to Assess the Prevalence of Hypertension and Its Associated Disorders among School Teachers in Dhaka City" showed that Hypertension is common among urban school teachers. Creating awareness by health education, healthy life style and behavior are necessary for prevention and control of hypertension among school teachers are necessary.

Review article on "What is new in new generation calcium channel blocker" reports the advantage of clinidipine for less reflex tachycardia, less pedal edema, less damage to glomeruli and suppress podocyte injury, better control of edema in comparison to other drugs in hypertension management.

Case Report on "Systemic Juvenile Idiopathic Arthritis (sJIA) or Still's Disease: A Diagnostic Challenge; while Presented as Fever of Unknown Origin. 2 Cases" emphasizes the need for considering Stills disease in case of fever of unknown origin in patients having fever, extra articular features as spiking fever, hepatosplenomegaly, lymphadenopathy, pleurisy, pericarditis and vasculopathy. Without any joint manifestations.
Case report on "Acardiac Acephalus Twin Pregnancy"emphasizes the need for early diagnosis of acardiac twin development to prevent increased mortality form this condition.

Case report on "Sarcoidosis - A Case Report" put emphasis on suspected cases with weight loss, irregular fever, skin rash, polyarthalgia, bodyache which are common symptoms for Tuberculosis, malignancy to bear in mind also for Sarcoidosis . Early diagnosis can lead to a better diagnosis and management.

Case report on "Dengue Typhoid Co-infection: a New Threat" emphasizes the need for correct diagnosis of dual infection in any febrile patient. The present case also states significant difficulty faced by clinicians to diagnose \& manage the case. The clinicians should understand the importance of early correct diagnosis is essential to mange this dual infection properly.

Hopefully this issue will also be enjoyable and informative to the valuable readers.

Prof. Dr. Md. Tahminur Rahman

MBBS, M. Phil, PhD

Editor in Chief, AKMMC Journal 